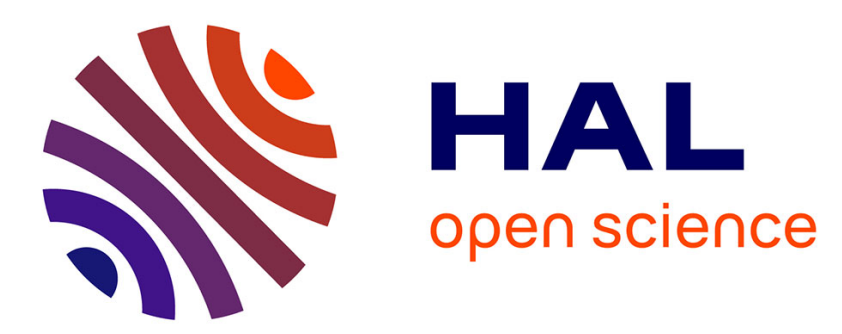

\title{
Excitation dynamics of micro-structured atmospheric pressure plasma arrays
}

H Boettner, J Waskoenig, D O ' Connell, J Winter, V Schulz-von Der Gathen

\section{To cite this version:}

H Boettner, J Waskoenig, D O ' Connell, J Winter, V Schulz-von Der Gathen. Excitation dynamics of micro-structured atmospheric pressure plasma arrays. Journal of Physics D: Applied Physics, 2010, 43 (12), pp.124010. 10.1088/0022-3727/43/12/124010 . hal-00569560

\section{HAL Id: hal-00569560 \\ https://hal.science/hal-00569560}

Submitted on 25 Feb 2011

HAL is a multi-disciplinary open access archive for the deposit and dissemination of scientific research documents, whether they are published or not. The documents may come from teaching and research institutions in France or abroad, or from public or private research centers.
L'archive ouverte pluridisciplinaire HAL, est destinée au dépôt et à la diffusion de documents scientifiques de niveau recherche, publiés ou non, émanant des établissements d'enseignement et de recherche français ou étrangers, des laboratoires publics ou privés. 


\title{
Excitation dynamics of micro-structured atmospheric pressure plasma arrays
}

\author{
H Boettner ${ }^{1}$, J Waskoenig ${ }^{2}$, D O'Connell ${ }^{2}$, J Winter ${ }^{1}$ and V \\ Schulz-von der Gathen ${ }^{1}$ \\ ${ }^{1}$ Institute for Experimental Physics II: Applied Plasma Physics, Ruhr-Universität \\ Bochum, 44780 Bochum, Germany \\ ${ }^{2}$ Centre for Plasma Physics, Queen's University Belfast, BT7 1NN Northern Ireland, \\ United Kingdom \\ E-mail: Henrik.Boettner@rub.de
}

\begin{abstract}
The spatial dynamics of the optical emission from an array of 50 times 50 individual micro cavity plasma devices are investigated. The array is operated in argon and argon-neon mixtures close to atmospheric pressure with an AC voltage. The optical emission is analysed with phase and space resolution. It has been found that the emission is not continuous over the entire AC period, but occurs once per half period. Each of the observed emission phases shows a self-pulsing of the discharge, with several bursts of emission of fixed width and repetition rate. The number of emission bursts depends on applied voltage and frequency. Spatially resolved measurements prove that the emission bursts are formed by overlapping emission pulses from single discharge cavities. Intensity differences between positive and negative half-wave can be interpreted through spatially resolved measurements of single discharge cavities.
\end{abstract}

Keywords: plasma diagnostics, plasma source, Townsend discharge, dielectric barrier discharge, microplasma, array, atmospheric pressure

PACS numbers: $52.25 . \mathrm{Os}, 52.50 . \mathrm{Dg}, 52.80 . \mathrm{Dy}, 52.70 . \mathrm{Kz}$

Submitted to: J. Phys. D: Appl. Phys.

\section{Introduction}

Recently microdischarge devices operated at atmospheric pressure enjoy increased attention; primarily due to the significant cost reduction for processing compared to their low pressure counterparts. So-called micro-structured electrode devices [1], including microplasma arrays [2], represent a sub-category of these discharges. Such devices promise the added advantage of homogeneous large area treatment. The microplasma arrays investigated in this work consisting of huge amounts of single micro plasma discharges are well suited for a variety of applications, e.g. plasma display panels (PDPs), photo-detectors, UV-sources, etc. [3, 4]. 
Electrical characteristics of such arrays have been well described by current voltage measurements as well as e.g. the integral optical efficiency $[3,5,2]$. Experimental investigations of the composing single discharge cavities are challenging, primarily due to the small dimensions and the operating conditions close to atmospheric pressure. A first approach to model a single microdischarge of an array operated in DC mode has been made by M. Kushner [6]. Although this model provides a good picture of a single microdischarge device, the interaction of several devices within an array and the effect of AC operation have not yet been considered. There are still many open questions concerning in particular the fundamental physics of the ignition phase, sustaining mechanisms and interaction of the single discharge cavities.

\section{Experimental setup}

In the present study we report on phase resolved optical emission spectroscopy (PROES) measurements on a microplasma array consisting of 50 times 50 single inverse pyramidal cavities etched into a silicon wafer, referred to as 'pixels'. These investigations offer technically easy access to time-dependent ignition and sustaining mechanisms. Each pixel has a $50 \mu \mathrm{m}$ squared base, $35 \mu \mathrm{m}$ depth and is coated with an $0.8 \mu \mathrm{m} \mathrm{Si}_{3} \mathrm{~N}_{4}$ dielectric. A $0.2 \mu \mathrm{m}$ thick nickel grid, coated with a $\mathrm{Si}_{3} \mathrm{~N}_{4}$ layer as well, serves as counter electrode. Both electrodes are additionally separated by a $10 \mu \mathrm{m}$ polyimide or $\mathrm{SiO}_{2}$ dielectric. A detailed description of the device can be found in publications of Eden et al. [7, 2]. The array is mounted on an adjustable post within a vacuum chamber with glass windows to provide a defined atmosphere (see figure 1). Here, it is operated in either pure argon or a mixture of neon and argon at a ratio of 4:1 close to atmospheric pressure (500 - 1000 mbar). The silicon base is grounded and the nickel grid is powered by a bipolar triangular voltage waveform with amplitudes up to $400 \mathrm{~V}$ and frequencies between 2 and $20 \mathrm{kHz}$. The voltage waveform is generated by a frequency generator (Tektronix AFG3021B) with subsequent broadband voltage amplifier (FM Elektronik DCU 600-40 HF). The triangular waveform allows to keep dV/dt constant in each half period. To monitor the emission spectrum of the microplasma array an USB grating spectrometer (Ocean Optics HR 4000CG-UV-NIR; resolution: $\sim 0.5 \mathrm{~nm}$ $F W H M)$ is used. For spatially integrated, time-dependent measurements of the emission a photomultiplier tube (PMT: Hamamatsu R3896) is mounted in front of the window facing the array. To obtain additional spatial information a repetitively gateable intensified charge-coupled device $(I C C D)$ camera (LaVision Picostar HR) is used alternatively. An optical system magnifies the array by a factor of 2 onto the sensitive chip of the camera $\left(13.2 \times 8.8 \mathrm{~mm}^{2}, 576 \times 384\right.$ pixels $)$. This yields a spatial resolution of $15 \mu \mathrm{m}$ per camera pixel. The camera gate is synchronised to the phase of the excitation frequency. Due to the available repetition rate of $100 \mathrm{MHz}$ of the camera every AC cycle can be used for integration. For phase resolution within the AC cycle measurements are taken every $200 \mathrm{~ns}$ with a gate interval of $200 \mathrm{~ns}$ for light collection. The emission is integrated over several cycles for good signal-to-noise ratio; the total integration time 
used in this work is approximately one second. Detailed descriptions of the principle of PROES using an ICCD can be found in the literature [8, 9]. Spectral discrimination by means of e.g. a spectrograph or a filter is applied neither for PMT measurements nor for phase and space resolved measurements using the ICCD camera. Commercial current (Tektronix P6021) and voltage (Tektronix P6015A) probes are installed to monitor the discharge during the measurements.

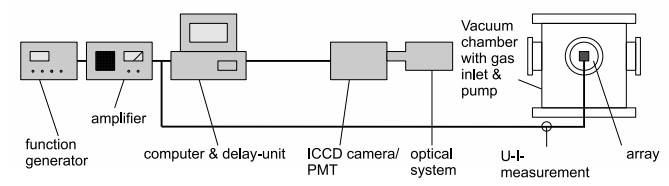

Figure 1. Sketch of the experimental setup

\section{Experiments}

\subsection{Discharge mode}

From its design and an electrical excitation (frequency, voltage) point-of view the microplasma array can be expected to behave similar to dielectric barrier discharges $(D B D s)$. Two discharge regimes are discussed for these DBDs, operation in glow and Townsend mode [13]. At elevated pressure and operation in noble gases these devices can be observed to operate in a so called 'glow' or 'pseudo-glow' regime and are in that case characterised as atmospheric pressure glow discharges $(A P G D)[10,11,12]$. Differing from the microplasma array these DBD's are typically set up in plane-parallel geometry with electrode gaps of $\mathrm{mm}$ dimension and diameters of several centimetres.

To characterise the discharge regime of the microplasma array emission line intensities of the Ne $2 \mathrm{p}_{1}$ and Ar $2 \mathrm{p}_{1}$ energy levels (Paschen notation) were measured using the USB grating spectrometer. The emission line intensity ratios of the neon $2 \mathrm{p}_{1}$ $\left(2 p_{1} \rightarrow 1 s_{2} ; \lambda=585.25 \mathrm{~nm}\right)$ transition and of the argon $2 \mathrm{p}_{1}\left(2 p_{1} \rightarrow 1 s_{2} ; \lambda=750.47 \mathrm{~nm}\right)$ transition were compared under pressure variation. Both energy levels can be assumed to be solely excited out of the ground state by electron impact excitation. While the electron impact excitation cross-section of Ar $2 \mathrm{p}_{1}$ [14] shows a negative slope, that of Ne $2 \mathrm{p}_{1}[15]$ shows a positive slope in the energy range between threshold and $50 \mathrm{eV}$. Hence, the evolution of the ratio of the respective $\mathrm{Ne} 2 \mathrm{p}_{1}$ and $\operatorname{Ar} 2 \mathrm{p}_{1}$ emission intensities

$$
\alpha=I(N e 585.25 \mathrm{~nm}) / I(\text { Ar } 750.47 \mathrm{~nm})
$$

under pressure variation is a measure for a shift of the high energetic part of the electron energy distribution function $(E E D F)$. While large values of $\alpha$ are an indicator for a negative glow discharge, where most of ionisation and excitation are caused by the beam component, small values suggest a Townsend-like discharge regime. Collisional de-excitation (quenching) of the respective energy levels was considered by weighting 
the measured intensities with calculated effective de-excitation rates for the particular pressures. The collisional quenching coefficients for neon were estimated as suggested by Mahony et al. [16] to be five times that of argon [17].

The discharge was operated in the $4: 1$ neon:argon composition at $5 \mathrm{kHz}$ driving frequency $f_{A C}$ and ramp excitation with $U_{p p}=780 \mathrm{~V}$ peak-to-peak voltage. The pressure $p$ was varied from 600 to 1000 mbar in 50 mbar steps. With increasing pressure $\alpha$ decreases indicating a relative decrease of hot electrons as shown in figure 2. This is in good qualitative agreement with the findings of Kushner [6]. For DC operation in pure neon he compared electron impact ionisation rates of $\mathrm{Ne}^{+}$and $\mathrm{Ne}^{2+}$ instead of emission line intensities and found the same pressure behaviour of the discharge. According to his definition the discharge investigated in this article can thus be characterised as DBD discharge in Townsend mode. This is also consistent with the findings of other works [13] which predict Townsend behaviour for small discharge cavities in He. The small confining structures of single array pixels fulfil this requirement. For measurements performed in the frame of this article the plasma can therefore be assumed to operate in Townsend mode.

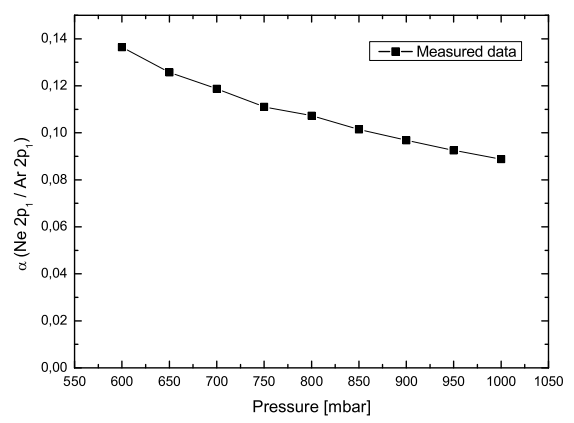

Figure 2. Ratio $\alpha$ of emission line intensities of $\mathrm{Ne}_{2} \mathrm{p}_{1}(585,33 \mathrm{~nm})$ and $\operatorname{Ar} 2 \mathrm{p}_{1}$ $(750,4 \mathrm{~nm})$ as measured with an USB grating spectrometer in a neon-argon mixture of $4: 1\left(f_{A C}=5 \mathrm{kHz}, U_{P P}=780 \mathrm{~V}\right) . \alpha$ characterises the discharge regime.

\subsection{AC excitation and self-pulsing}

$\mathrm{AC}$ frequency is an important parameter for the control of the discharge. To investigate the influence of driving frequency on the emission of the array, temporally, spatially and spectrally integrated intensities were recorded for different driving frequencies. For a constant gas pressure of 1000 mbar argon the intensity as well as the intensity normalised to the driving frequency as a function of driving frequency are illustrated in figure 3. While the overall intensity increases with increasing driving frequency, the intensity normalised to the frequency i.e. per cycle decreases. To understand this phenomenon, time resolved measurements were performed using the PMT.

The general time-dependent behaviour of the investigated microplasma arrays has already been described by Waskoenig et al. [18]. The spectrally integrated emission 


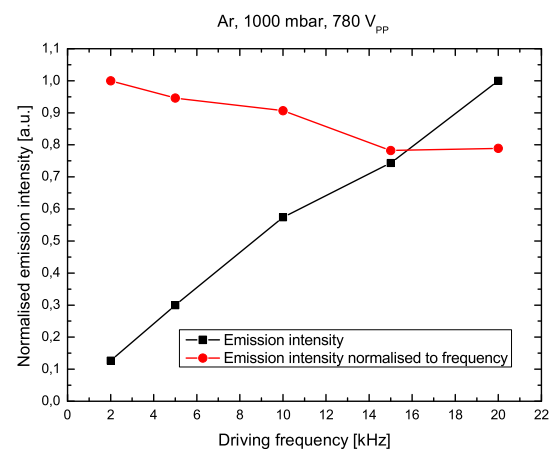

Figure 3. Normalised integrated emission intensity of the whole microplasma array as well as the latter normalised to the driving frequency. The data was measured using an USB grating spectrometer in pure argon $\left(p=1000\right.$ mbar, $f_{A C}=5 \mathrm{kHz}, U_{P P}=$ $780 \mathrm{~V}$.)

intensity of the microplasma array measured time resolved with a PMT is exemplarily shown in figure 4 for a gas mixture of neon and argon at a ratio of 4:1 and 1000 mbar pressure, $5 \mathrm{kHz}$ driving frequency and $780 \mathrm{~V}$ peak-to-peak voltage. After passing a certain threshold voltage $U_{0}$ a series of discharge bursts occurs until the vertex in the driving voltage is reached. Close to this point the electric field direction in the discharge reverses due to residual charge carriers adsorbed at the dielectric surfaces. This causes the series of bursts to abort until the increasing electric field in the reverse direction allows a new series of bursts of reverse polarity. The intensity of the peaks decreases continuously. The repetition rate of this self-pulsing lies in the range of a few hundred $\mathrm{kHz}$, while the pulse width is in the order of a few $\mu \mathrm{s}$. A similar behaviour was observed for plane-parallel dielectric barrier discharges with electrode areas of several square centimetres and electrode gaps of several hundred $\mu \mathrm{m}$ by other groups $[11,19,12]$. It has to be pointed out that in contrast to these discharges the array emission is composed out of the emission from several individual cavities [18]. Each observed burst itself consists of a wave-like ignition of all of the single microplasma pixels, which is reproducible. During one burst every pixel ignites once on a time scale one order of magnitude lower compared to the burst. Detailed analysis of single burst evolution can be found in the literature [18]. The self-pulsing of the discharge is another feature typical for DBDs in Townsend mode [13]. Figure 5 exemplarily shows the inhomogeneous emission intensity distribution of the microplasma array registered with the ICCD during the first burst of the positive half period for a gate interval of $200 \mathrm{~ns}$. The array was operated at 1000 mbar pressure, $10 \mathrm{kHz}$ driving frequency and $780 \mathrm{~V}$ peak-to-peak voltage.

The part of the excitation period when the nickel grid is on positive and negative potential respectively will be referred to as 'positive half period' and 'negative half period' in the further discussion of this work. An interesting aspect is a significantly weaker emission observed in the negative half period compared to the positive half period. This asymmetry of the burst emission intensity comparing both half periods 


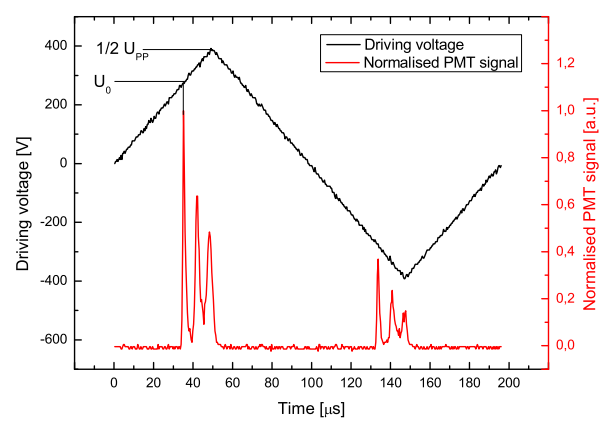

Figure 4. Normalised PMT signal and driving voltage measured for a neon-argonmixture at a ratio of 4:1 $\left(p=1000 \mathrm{mbar}, f_{A C}=5 \mathrm{kHz}, U_{P P}=780 \mathrm{~V}\right)$.

will be discussed later.

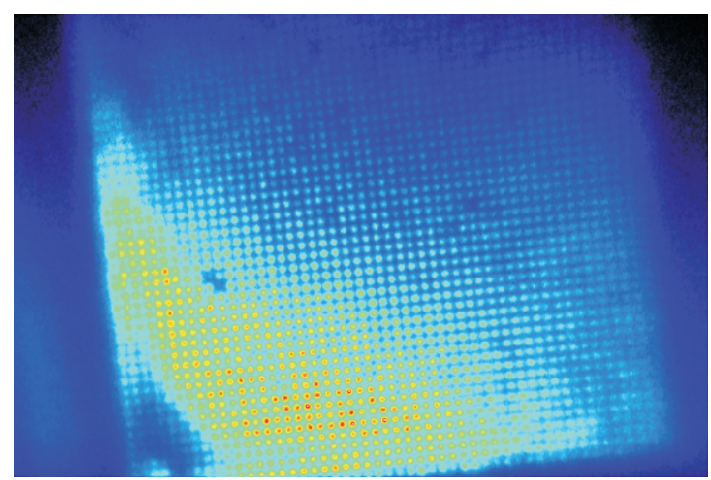

Figure 5. Spectrally integrated emission intensity distribution of the microplasma array as registered with the ICCD camera for a gate interval of $200 \mathrm{~ns}(p=1000 \mathrm{mbar}$, $\left.f_{A C}=10 \mathrm{kHz}, U_{P P}=780 \mathrm{~V}\right)$. Intensity scaling from minimum (blue) to maximum $(r e d)$.

As also observed by Radu et al. for plane-parallel DBDs [11] the number of bursts per half period is dependent on driving frequency and voltage. To quantify this phenomenon for the investigated microplasma array the discharge was operated in pure argon at a pressure of 1000 mbar. While the driving peak-to-peak voltage was kept constant at $780 \mathrm{~V}$, the driving frequency was varied from $2 \mathrm{kHz}$ to $20 \mathrm{kHz}$. Voltages of initial breakdowns $U_{0}$ were determined at the peak of the corresponding emission maximum registered with the PMT. As illustrated in figure 6 the number of bursts per half period decreases with increasing driving frequency. In case the last peak of a burst did not develop completely it was counted half. Measurements in the neon-argon mixture yield comparable results. This explains the decrease of normalised intensity with increasing driving frequency observed earlier.

Moreover, the initial breakdown voltage increases with increasing driving frequency from $300 \mathrm{~V}$ to $350 \mathrm{~V}$. This behaviour can be understood using a simple model for the breakdown of the discharge. The voltage over the discharge gap is decreased by charge 


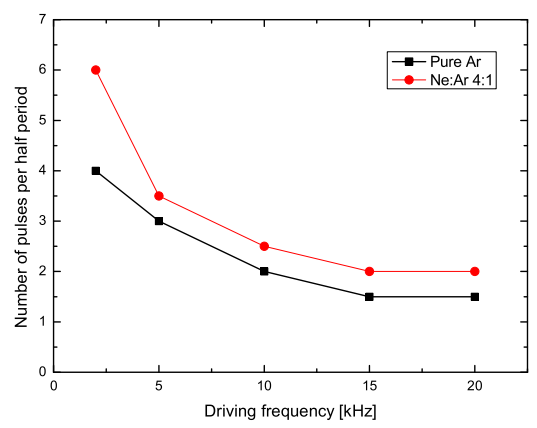

Figure 6. Number of bursts per half period as a function of driving frequency as measured with a PMT in pure argon and a neon-argon mixture at a ratio of 4:1 $(p=$ 1000 mbar, $\left.U_{P P}=780 \mathrm{~V}\right)$. Bursts cut off are counted half.

carriers that follow the induced electric field and adsorb at the dielectric layers on the electrodes. Here, the transit time of the ions to cross the discharge can be seen as a characteristic time $t_{D}$. Due to the comparably high ion inertia, the time-determining factor is the ion drift velocity $v_{D}$. To overcome the reduction of the discharge voltage an increased applied voltage $U_{0}$ is necessary to reach the breakdown voltage $U_{B}$ over the discharge gap $d$. Then $U_{B}$ is given by

$$
U_{B}=U_{0}-\frac{d U}{d t} t_{D}
$$

where

$$
t_{D}=\frac{d}{v_{D}}=\frac{d^{2}}{\mu_{i}\langle U\rangle},\langle U\rangle=\frac{U_{0}+U_{B}}{2}
$$

and $\mu_{i}$ is the ion mobility. Substitution of driving frequency for the derivative gives

$$
U_{0}^{2}=U_{B}^{2}+\frac{4 U_{P P} d^{2}}{\mu_{i}} f_{A C}
$$

The squared applied voltages $U_{0}$ for the first breakdown in the positive half period are shown in figure 7 as a function of the driving frequency for operation in pure argon at 1000 mbar pressure and $780 \mathrm{~V}$ peak-to-peak voltage. Curvilinear streamlines where assumed for the inter-electrode gap $d$ to take into account the pyramidal geometry. Assuming an effective $d=60 \mu \mathrm{m}$, a linear fit of the data points according to equation (4) yields $U_{B}=(300 \pm 7) \mathrm{V}$.

For the gas mixture under otherwise identical conditions measurements yield a breakdown voltage of $U_{B}=(290 \pm 5) \mathrm{V}$ lying below that determined for pure argon. This can be explained by higher ion mobilities in case of the gas mixture. The linear regression of the measured data points in figure 7 yields a value of $\sim 2$ for the ratio of mean ion mobilities in the neon-argon gas mixture and that in pure argon. This corresponds very well with the ratio deduced from tabulated ion mobilities $[20,21,22]$ for mean reduced electric fields of $\mathrm{E} / \mathrm{N} \leq 50 \mathrm{Td}$.

The increased driving voltage required to reach the breakdown voltage with increasing driving frequency also explains the reduction of the number of bursts per 


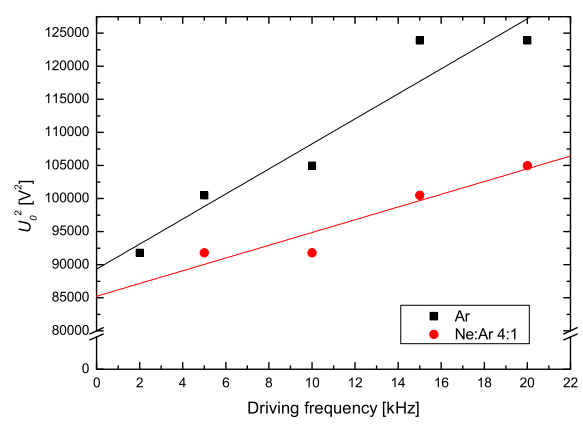

Figure 7. Squared applied voltages $U_{0}$ for the first breakdown in the positive half period as a function of driving frequency measured in pure argon and a 4:1 neon-argon mixture $\left(p=1000\right.$ mbar, $\left.U_{P P}=780 \mathrm{~V}\right)$. Lines represent respective linear regressions. According to equation (4) these yield breakdown voltages $U_{B}$.

half period. At increased driving frequencies the time interval between breakdown voltage and vertex of the applied driving voltage gets shorter. Thus, less time is left for subsequent bursts, especially as the ion mobility as time determining factor for burst evolution is invariant towards frequency variation.

The number of bursts per half period can be influenced by variation of the driving peak-to-peak voltage as well. To verify this correlation the microplasma array was operated in the neon and argon mixture at a pressure of 1000 mbar and a driving frequency of $5 \mathrm{kHz}$. While the driving voltage was increased from 640 to $820 \mathrm{~V}$, the number of bursts per half period increased from one to four.

Comparison of the potential difference between a vertex of the driving voltage and the peak of the first burst in the following half period showed the latter to be invariant towards a variation of the driving peak-to-peak voltage. The first discharge burst in the negative half period constantly evolves after a voltage change of about $(670 \pm 10) \mathrm{V}$ after the vertex. The value is $\sim 5 \%$ higher for the positive half period. This asymmetry will be discussed later. Thus, an increase of the applied peak-to-peak voltage increases the time between the first burst and the subsequent vertex. As the frequency of the bursts is independent of the applied peak-to-peak voltage, this allows more bursts to ignite. An increase of the applied peak-to-peak voltage consequently has a similar effect on the pulsing behaviour of the discharge as a decrease of the driving frequency.

\subsection{Collective and individual pixel behaviour}

As already discussed the array does not ignite simultaneously but shows repetitive spatial variations similar to wave structures [18]. To compare single pixel to collective array behaviour, the spectrally integrated optical emission of the discharge was recorded for the first half period of the driving voltage phase and space resolved with the ICCD camera as well as temporally resolved with a PMT. The array was operated in pure argon at 1000 mbar pressure, $10 \mathrm{kHz}$ driving frequency and $780 \mathrm{~V}$ peak-to-peak voltage. 
Results are shown in figure 8. The PMT measurements yielded an FWHM of $\sim 1.2 \mu$ s for the first emission peak resulting from the first discharge burst in the positive half period. At the same time PROES allowed to observe the temporal evolution of the emission intensity for one single cavity. Here, an FWHM of $\sim 0.3 \mu$ s was derived. Thus, the burst duration is approximately one order of magnitude higher for the whole microplasma array compared to single cavities. This is consistent with the observation of the wavelike ignition of the single pixels per emission burst.

These results can be qualitatively compared with calculations using a onedimensional fluid model for plane-parallel homogeneous DBDs operated in helium by Golubovskii et al. [13]. This model amongst others allows to calculate the oscillation frequency of discharge bursts similar to those observed in this work. Necessary input parameters are the discharge dimension, thickness of the dielectric layer, growth rate of the external voltage, ion mobility, electric field strength and derivative of the first Townsend coefficient for the gas used with respect to the electric field strength. Using the model for an approximation of the discharge behaviour of the microplasma array investigated in this work gave results in relatively good agreement with PROES and spectroscopic measurements performed.

For a significant discharge dimension of $60 \mu \mathrm{m}$, a thickness of $0.8 \mu \mathrm{m}$ of the dielectric layer, an electric field strength of $\leq 110000 \mathrm{Vcm}^{-1}$, a driving frequency of $5 \mathrm{kHz}$ and a peak-to-peak voltage of $780 \mathrm{~V}$ an oscillation frequency of a few $\mathrm{MHz}$ and a period time of a few $0.1 \mu$ s respectively are calculated. This corresponds well with the experimental results for a single discharge cavity. Assuming a discharge dimension of $5000 \mu \mathrm{m}$ according to the whole array dimension yields a periodic time of a few $\mu \mathrm{s}$ which approximates the FWHM of the observed emission of the whole array quite well. Of course, the model used here does neither account for the pyramidal geometry of the single cavities nor for the array-structure.

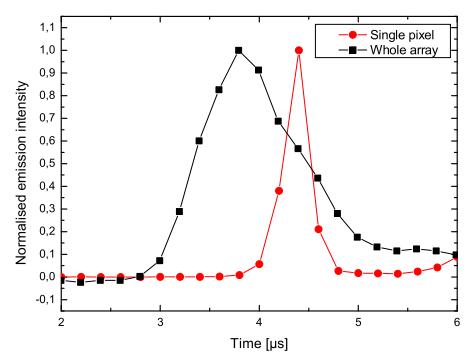

Figure 8. Normalised emission intensity of the first burst measured with the PMT for the whole array and the ICCD camera for one single pixel in a pure argon discharge $\left(p=1000 \mathrm{mbar}, f_{A C}=10 \mathrm{kHz}, U_{P P}=780 \mathrm{~V}\right)$.

As also observed in previous works [18] the microplasma arrays investigated here show an asymmetric behaviour comparing the positive and negative half period. Although the number of bursts, their frequency and the time between vertex of the driving voltage and the first burst are the same for both half periods, the spectrally 
integrated emission intensity of single bursts is smaller in the negative half period (see figure 4). This feature is due to the asymmetry of the electrodes in the devices.

Phase resolved imaging of the spectrally integrated optical emission of the microplasma array in operation showed brighter and more spacious emission from single microplasma cavities in the positive compared to the negative half period. This effect could be observed independent of gas mixture, pressure, driving frequency or peak-topeak voltage. Apart from geometrical asymmetry of the electrodes it can be understood as most excitation leading to the observed emission features is due to electron-impact excitation. For Townsend discharges the emission maximum is observed close to the anode, due to the localised highest electron density and energy respectively. In the positive half period electrons are accelerated out of the microplasma cavities towards the Ni electrode. They reach the excitation threshold at the top of the single cavities leading to a spacious emission feature. In the negative half period electrons are accelerated from the $\mathrm{Ni}$ electrode into the microplasma cavities, reaching the excitation threshold deeper inside. In this direction more electrons can get lost due to surface neutralisation so that less electrons can reach the threshold. Thus, less species are excited in a smaller volume and a more confined and less bright emission feature is observed.

Figure 9 exemplarily shows images of the spectrally integrated emission intensity densities integrated over the corresponding half period from PROES measurements. The array was operated in pure argon at 1000 mbar pressure, $10 \mathrm{kHz}$ driving frequency and $780 \mathrm{~V}$ peak-to-peak voltage. Beneath, presumed electron trajectories indicated by arrows and the resulting emission features inside a single microplasma cavity are sketched for both half periods.

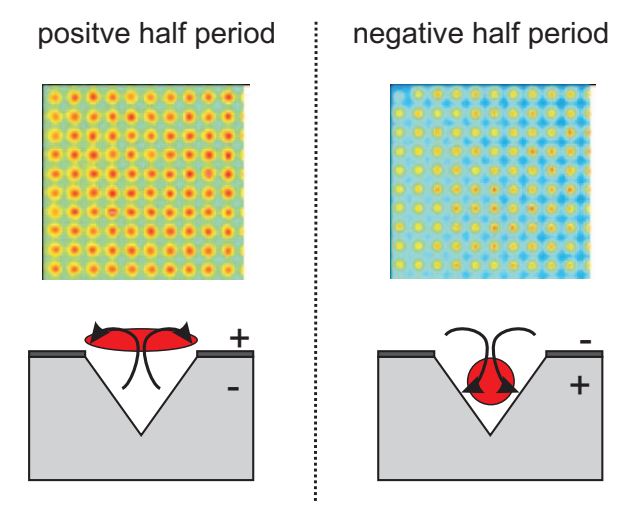

Figure 9. Spectrally integrated emission intensity of a section of the microplasma array as recorded in pure argon with the ICCD camera (false colours, scaling from zero counts (blue) to maximum (red)) for the positive and negative half period respectively $\left(p=1000\right.$ mbar, $\left.f_{A C}=10 \mathrm{kHz}, U_{P P}=780 \mathrm{~V}\right)$.

\section{Conclusion}

Space and phase resolved investigation of the emission of micro arrays with inverse pyramidal configuration shows a behaviour very similar to that observed for 
homogeneous dielectric barrier discharges with plane-parallel geometry operated under similar conditions. Those discharges have been observed to develop radial structures that expand over the discharge area or develop crystal-like structures. The lifetime of these single emission structures can be explained by models taking into account the ion-mobility between the electrodes. For the micro discharge arrays a similar characteristic emission time can be observed. The number of bursts per cycle is determined by driving AC frequency and peak-to-peak voltage. However, here the emission is composed of single emission peaks of much shorter lifetime of a few hundred nanoseconds emitted from each single cavity. The emission i.e. the discharge is then transferred to neighbouring cavities forming wave-like emission structures.

Intensity differences between the polarities of the applied voltage could be shown to be related to the asymmetry of the fields driving the exciting electrons.

A more detailed analysis of the interaction between neighbouring cavities and of the excitation processes within a single cavity is prepared by introducing an additional wavelength selector.

\section{Acknowledgments}

The authors would like to thank J. Gary Eden and Sung-Jin Park from the University of Illinois, Urbana-Champaign for the lively cooperation, supply of microplasma arrays and valuable discussions. This work is supported by the 'Deutsche Forschungsgemeinschaft' (DFG) within the frame of the research group 'FOR1123 - Physics of Microplasmas' and the research project 'SCHU2353/1-1', both part of the research department 'Plasmas with Complex Interactions', as well as by the 'Ruhr-University Research School'. One of the authors (DOC) gratefully acknowledges the support by an 'FP7 Marie Curie Intra-European Fellowship'.

\section{References}

[1] Baars-Hibbe L, Sichler P, Schrader C, Lucas N, Gericke K-H and Büttgenbach S 2005 J. Phys. D: Appl. Phys. 38 510-17

[2] Eden J G and Park S-J 2005 Plasma Phys. Control. Fusion 47 B83-92

[3] Eden J G, Park S-J, Ostrom N P and Chen K-F J. Phys. D: Appl. Phys. $381644-48$

[4] Park S-J, Eden J G and Ewing J J 2002 Appl. Phys. Lett. 81 4529-31

[5] Park S-J, Chen J, Wagner C J, Ostrom N P, Liu C and Eden J G 2002 IEEE J. Sel. Top. Quantum Electron. 8 387-94

[6] Kushner M J 2004 J. Appl. Phys. 95 846-59

[7] Park S-J, Chen J, Liu C and Eden J G 2001 Appl. Phys. Lett. 78 419-21

[8] Gans T, Schulz- von der Gathen V and Döbele H F 2004 Contrib. Plasma Phys. 44 523-28

[9] O'Connell D, Gans T, Vender D, Czarnetzki U and Boswell R 2007 Phys. Plasmas 14034505

[10] Radu I, Bartnikas R, Czeremuszkin G and Wertheimer M R 2003 IEEE Trans. Plas. Science 31 411-421

[11] Radu I, Bartnikas R and Wertheimer M R 2003 IEEE Trans. Plas. Science 31 1363-78

[12] Mangolini L, Orlov K, Kortshagen U, Heberlein J and Kogelschatz U 2002 Appl. Phys. Lett. 80 1722-24 
[13] Golubovskii Y B, Maiorov V A, Behnke J and Behnke J F 2003 J. Phys. D: Appl. Phys. 36 39-49

[14] Chilton J E, Boffard J B, Schappe R S and Lin C C 1998 Phys. Rev. A 57 267-77

[15] Chilton J E, Stewart M D, Jr. and Lin C C 2000 Phys. Rev. A 61052708

[16] Mahony C M O, Gans T, Graham W G, Maguire P D and Petrovic Z L 2008 Appl. Phys. Lett. 93 011501

[17] Francis A, Gans T, Niemi K, Czarnetzki U, Schulz-von der Gathen V and Döbele H F Proc. SPIE 4460122

[18] Waskoenig J, O'Connell D, Schulz-von der Gathen V, Winter J, Park S-J and Eden J G 2008 Appl. Phys. Lett. 92101503

[19] Sublet A, Ding C, Dorier J-L, Hollenstein Ch, Fayet P and Coursimault F 2006 Plasma Sources Sci. Technol. 15 627-34

[20] Ellis H W, Pai R Y, McDaniel E W, Mason E A and Viehland L A 1976 Atomic Data and Nuclear Data Tables 17 177-210

[21] Ellis H W, McDaniel E W, Albritton D L, Viehland L A, Lin S L and Mason E A 1978 Atomic Data and Nuclear Data Tables 22 179-217

[22] Ellis H W, Thackston M G, McDaniel E W and Mason E A 1984 Atomic Data and Nuclear Data Tables 31 113-151 